\title{
Determination of the high-strain rate elastic modulus of printing resins using two different split Hopkinson pressure bars
}

\author{
S. Aghayan ${ }^{1}$ (D) S. Bieler ${ }^{1} \cdot$ K. Weinberg ${ }^{1}$
}

Received: 19 April 2021 / Accepted: 26 July 2021 / Published online: 26 August 2021

(c) The Author(s) 2021

\begin{abstract}
The usage of resin-based materials for 3D printing applications has been growing over the past decades. In this study, two types of resins, namely a MMA-based resin and an ABSbased tough resin, are subjected to compression tests on a split Hopkinson pressure bar to deduce their dynamic properties under high strain rate loading.

Two Hopkinson bar setups are used, the first one is equipped with aluminum bars and the second one with PMMA bars. From the measured strain waves, elastic moduli at high strain rates are derived. Both setups lead to values of $E=3.4-3.8 \mathrm{GPa}$ at a strain rate of about $250 \mathrm{~s}^{-1}$. Numerical simulations support the experiments. Moreover, considering the waves gained from the two different bar setups, PMMA bars appear to be well-suited for testing resin samples and are therefore recommended for such applications.
\end{abstract}

Keywords Split Hopkinson pressure bar · 3D printing resin · PMMA SHPB · High-strain rate elastic modulus

\section{Introduction}

Polymeric resins are typical materials for additive manufacturing with a wide range of applications. While their static elastic properties are commonly determined in tension or bending tests, the behavior of such materials under dynamic loading is mostly unclear. Because of the intrinsic viscosity of the polymeric materials, their response under high speed loading is much stiffer than under slow deformation. The vast applications of additively printed materials, particularly regarding prototyping, repairs and replacements (Jandt and Sigusch 2009; Bonyar and Santha 2010; Ruamario and Sergio 2019; Zhao and Wang 2017), but also high

S. Aghayan

sam.aghayan@uni-siegen.de

S. Bieler

soeren.bieler@uni-siegen.de

K. Weinberg

kerstin.weinberg@uni-siegen.de

1 Lehrstuhl für Festkörpermechanik, Universität Siegen, Paul-Bonatz-Straße 9-11, 57076 Siegen, Germany 


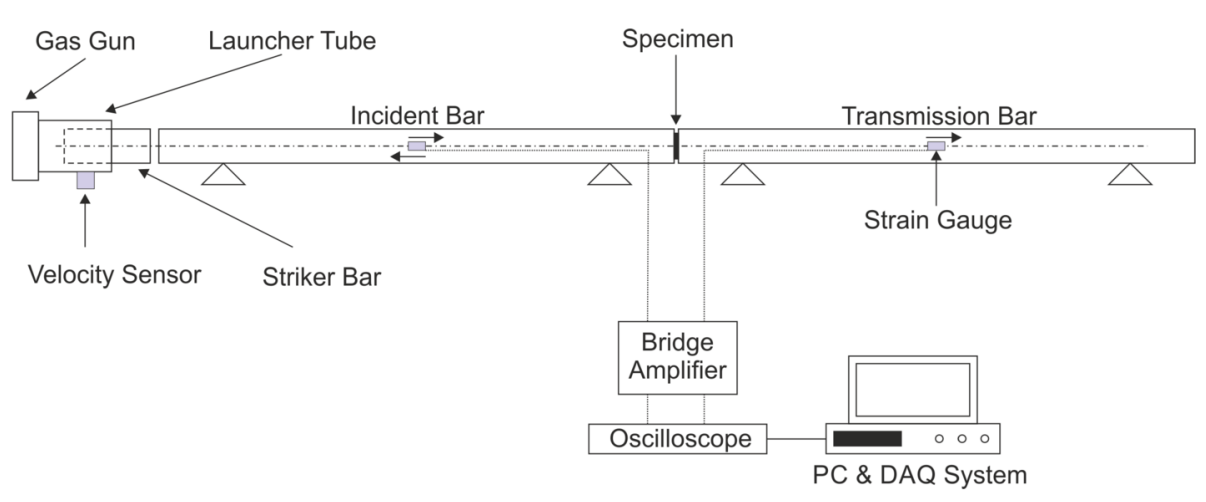

Fig. 1 Split Hopkinson pressure bar setup used for the compression tests

impact-resistant operations (Roberson and Perez 2015), encourage us to investigate this aspect more deeply. There have been previous investigations on the dynamic properties of resins in general (Sabbagh and Leloup 2002; Whiting and Jacobsen 1980; Jager and Balthazard 2016; Marghalani 2016; Ferracane and Pfeifer 2014), but only during recent years experiments on 3D printing resin-based materials have been reported (Hong and $\mathrm{Hu} 2021$; Rua and Buchely 2019).

The split Hopkinson pressure bar (SHPB), with a schematic diagram shown in Fig. 1, is a common experimental device to determine the dynamic material properties, mainly capable of subjecting the material to moderate and high strain rates (Kolsky 1963; Chen and Song 2011; Owens and Tippu 2009; Weinberg et al. 2018; Weinberg and Khosravani 2018; Hopkinson 1914; Chen and Zhou 1998; Zhao and Knauss 2007). Because resins are soft materials with a rather high impedance, two SHPB setups are considered in this study. In the first setup, the split Hopkinson bar is equipped with classical aluminum bars and in the second setup the bars are made of PMMA material. With both SHPB setups we test and evaluate two thermoplastic polymers of additive manufacturing.

In this study, the elastic modulus at high strain rates, typically referred to as dynamic elastic modulus in SHPB tests, of two printing resins are investigated. The two materials investigated are: a standard printing resin which is a white, methyl methacrylate (MMA) based plastic and a thermoplastic resin which corresponds to acrylonitrile butadiene styrene (ABS). Both materials and the general experimental setup are described in Sect. 2. Technical details, including principals and assumptions of the SHPB test, are explained in Sect. 3. In Sect. 4 the obtained results for the two SHPB setups are presented, discussed and evaluated. A short summary is given in Sect. 5 .

\section{Specimens and experimental setup}

The specimens tested in our SHPB experiments are engineering resins produced and provided by the company Formlabs (Formlabs 2020) for various 3D printing purposes. The specimens were printed by the SLA 3D printer (Formlabs) with high precision. After the printing process the samples were polished. Hence, the materials are considered to be homogeneous and isotropic throughout the test. Both are thermoplastic polymers but have different base materials and therefore different mechanical specifications. The classical standard 
Table 1 Properties of the investigated 3D printing materials (Formlabs 2020)

\begin{tabular}{llllll}
\hline Resin type & $\begin{array}{l}\text { Basis } \\
\text { polymer }\end{array}$ & $\begin{array}{l}\text { Tensile } \\
\text { modulus } \\
{[\mathrm{GPa}]}\end{array}$ & $\begin{array}{l}\text { Flexural } \\
\text { modulus } \\
{[\mathrm{GPa}]}\end{array}$ & $\begin{array}{l}\text { Notched } \\
\text { IZOD } \\
{[\mathrm{N} / \mathrm{m}]}\end{array}$ & Remark \\
\hline $\begin{array}{l}\text { White standard } \\
\text { resin (SR) } \\
\begin{array}{l}\text { Blueish tough } \\
\text { resin (TR) }\end{array}\end{array}$ MMA & 2.8 & 2.2 & 25 & $\begin{array}{l}\text { high surface and } \\
\text { dimensional quality } \\
\text { sturdy prototyping } \\
\text { high durability }\end{array}$ \\
\hline
\end{tabular}

resin (SR) is a strong acrylic plastic of matte white tone. The tough resin (TR) is ABSbased, with some flexibility and a comparatively high fracture resistance. The mechanical properties of the SR and TR resins are shown in Table 1.

The geometry of the specimens for the compression SHB tests is cylindrical with a diameter of $18 \mathrm{~mm}$. For the setup with aluminum bars, the samples have a length of $9 \mathrm{~mm}$. Thus, the length-to-diameter ratio is 0.5 , which is favorable to minimize inertia effects. Moreover, rather thin specimens are needed to facilitate the dynamic stress equilibrium (Dai and Huang 2010; Gorham 1989). For the PMMA bars the length of the samples are $15 \mathrm{~mm}$. Here we need longer specimen in order to obtain the same strain rate.

The SHPB setup consists of a striker, accelerated by a gas gun, an incident and a transmission bar (Kolsky 1963; Chen and Song 2011). The specimen is placed between the bars. When the striker hits the incident bar, a compressive pulse is generated and at the barspecimen interface the wave splits, a part is reflected and a part continues to propagate through the specimen and the transmission bar; see Fig. 1. The pulses are determined by the materials' susceptibility to wave propagation, i.e. the acoustic impedances $z=A \rho c$ where $A, \rho$ are cross section and material density, respectively, and $c$ is the wave speed. At the interface of two materials the corresponding wave portions are (Chen and Song 2011)

$$
\sigma_{T}=\frac{2 z_{2}}{z_{1}+z_{2}} \frac{A_{1}}{A_{2}} \sigma_{I}, \quad \sigma_{R}=\frac{z_{2}-z_{1}}{z_{1}+z_{2}} \sigma_{I} .
$$

The difference in acoustic impedances of aluminum bars and polymer specimen may result in an insufficient transmitted pulse. When the impedance difference is very large $\left(z_{2} \gg z_{1}\right)$ almost the entire wave is reflected at the interface of the incident bar and the specimen. As a result, only a weak or no impulse can be measured at the transmission bar. This holds true, in particular, for soft materials. The tested SR and TR materials have impedances of 0.7 and $0.6 \mathrm{~kg} / \mathrm{m}^{2} \mu \mathrm{s}$, respectively. To reduce the impedance mismatch between specimen and bars, PMMA as bar material has been considered. In general, PMMA bars are suitable for testing softer materials. For resins with high impedance, aluminum bars are sufficient to obtain both a transmitted signal and a reflected signal. For soft resins, PMMA bar would be the only suitable choice to obtain a transmitted pulse. Details of the used PMMA bars are given in Table 2 .

To record the wave data two strain gauges with a resistance of $120.0 \pm 0.1 \Omega$ and a sensitivity coefficient of $2.14 \pm 1 \%$ are appliqued in the middle of each bar.

\subsection{Aluminum SHPB bar setup}

The first SHPB setup uses aluminum (EN AW-6061) incident and transmission bars with parameters of Table 2. Aluminum cylindrical strikers, being made of the same aluminum 
Table 2 Dimensions and mechanical properties of aluminum and PMMA bars

\begin{tabular}{lll}
\hline & Aluminum bars & PMMA bars \\
\hline Length $[\mathrm{mm}]$ & 1800 & 1800 \\
Diameter $[\mathrm{mm}]$ & 20 & 20 \\
Static elastic modulus $[\mathrm{GPa}]$ & 70 & 3.5 \\
Density $\left[\mathrm{kg} / \mathrm{m}^{3}\right]$ & 2700 & 1178 \\
Wave speed $[\mathrm{m} / \mathrm{s}]$ & 5052 & 1716 \\
Impedance $\left[\mathrm{kg} / \mathrm{m}^{2} \mu \mathrm{s}\right]$ & 13.64 & 2.02 \\
\hline
\end{tabular}

Fig. 2 Tough resin sample fixed between PMMA bars

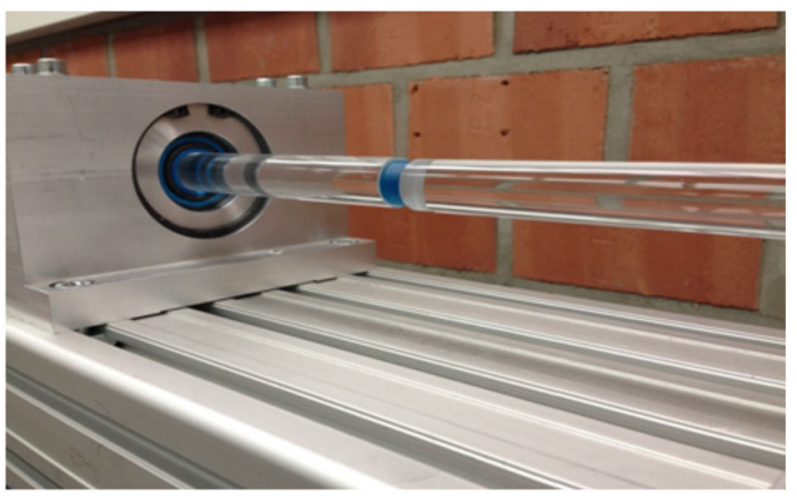

with $20 \mathrm{~mm}$ diameter and length of $100 \mathrm{~mm}$, are used. The mass of the aluminum striker is then $0.084 \mathrm{~kg}$. The striker is accelerated by the gas gun, which results in a striker velocity of almost $10 \mathrm{~m} / \mathrm{s}$. From Eq. (1) we calculate for aluminum bars and a SR specimen, transmitted wave portions of $\sigma_{T} / \sigma_{I}=0.1$ which illustrates the problem of a high impedance mismatch.

\subsection{PMMA SHPB bar setup}

The second SHPB setup is equipped with the PMMA bars of the same length and diameter; see Fig. 2. The corresponding cylindrical PMMA striker has also a diameter of $20 \mathrm{~mm}$, a length of $100 \mathrm{~mm}$ and a mass of $0.037 \mathrm{~kg}$; its acceleration results in a velocity of $10 \mathrm{~m} / \mathrm{s}$. Since the PMMA bars tend to deform due to the impact, the striker velocity has to be calibrated carefully. For the PMMA bars and a SR specimen we calculate from Eq. (1) transmitted wave portions of $\sigma_{T} / \sigma_{I}=0.6$. This corresponds to our observation of the transmitted pulse being significantly higher when using PMMA bars, compared to aluminum bars.

\section{Prerequisites of SHPB testing}

The SHPB experiment is based on one-dimensional wave theory. The induced longitudinal wave $u(x, t)$, and its parts after reflection and transmission, are measured as strain signals $\epsilon_{I}(t), \epsilon_{T}(t), \epsilon_{R}(t)$, where the indices $I, R$ and $T$ refer to the incident, reflected and transmitted wave accordingly. The displacement at the specimen-incident-bar interface $u_{1}$ and the specimen-transmission-bar interface $u_{2}$ can be derived from the measured strain signals 
(Kolsky 1963; Chen and Song 2011)

$$
u_{1}=c_{b} \int_{0}^{t}\left(\epsilon_{I}-\epsilon_{R}\right) \mathrm{d} t, \quad u_{2}=c_{b} \int_{0}^{t} \epsilon_{T} \mathrm{~d} t
$$

The indices $b$ and $s$ refer to bar and specimen, respectively. The strain in the specimen with the length $L_{s}$ can be calculated by

$$
\epsilon_{s}=\frac{u_{2}-u_{1}}{L_{s}}=\frac{c_{b}}{L_{s}} \int_{0}^{t}\left(\epsilon_{I}-\epsilon_{R}-\epsilon_{T}\right) \mathrm{d} t .
$$

From Hooke's law the axial stresses and the corresponding axial forces are derived. The reaction forces $F_{1}=F_{I}-F_{T}$ and $F_{2}=F_{T}$ result as

$$
F_{1}=E A_{b}\left(\epsilon_{I}+\epsilon_{R}\right), \quad F_{2}=E A_{b}\left(\epsilon_{T}\right) .
$$

Averaging these forces and dividing by area $A_{s}$ give the stress in the specimen

$$
\sigma_{s}=\frac{E_{b} A_{b}}{2 A_{s}}\left(\epsilon_{I}+\epsilon_{R}+\epsilon_{T}\right)=E_{b} \frac{A_{b}}{A_{s}} \epsilon_{T} .
$$

Then the strain in the specimen and the strain rate result as

$$
\begin{aligned}
& \epsilon_{s}=-2 \frac{c_{b}}{L_{s}} \int_{0}^{t} \epsilon_{R} \mathrm{~d} t, \\
& \dot{\epsilon}_{s}=-2 \frac{c_{b}}{L_{s}} \epsilon_{R},
\end{aligned}
$$

and the dynamic elastic modulus of the specimen is obtained by

$$
E_{\mathrm{dyn}}=\frac{\sigma_{s}}{\epsilon_{s}}=-\frac{A_{b} E \epsilon_{T} L_{s}}{2 A_{s} c_{b} \int_{0}^{t} \epsilon_{R} \mathrm{~d} t} .
$$

\subsection{Stress equilibrium}

The SHPB experiment is based on two fundamental assumptions: The first one is the assumption of one-dimensional wave theory, which is related to the calibration of the setup. The bars in the SHPB test must be well-aligned so that the stress wave propagates without significant lateral effects and without dispersion. For the SHPB test in this study, a laser technique was utilized in order to adjust the proper bar alignment. The second assumption is the stress equilibrium condition, which is related to an instantaneous equilibrium of forces in the loaded specimen, $F_{1} \approx F_{2}$, Equation (4). This assumption is basically related to an axially uniform state of deformation and needs to be examined during the test. To do so, we use here a strategy suggested in Ravichandran and Subhash (1994) and determine a parameter relating the forces Eq. (4) at the specimen-incident bar interface and the specimen-transmission bar interface, respectively;

$$
R(t)=\frac{\Delta F(t)}{F_{\text {avg }}(t)}=2\left|\frac{F_{1}(t)-F_{2}(t)}{F_{1}(t)+F_{2}(t)}\right| .
$$

The specimen is in stress equilibrium when $R(t)$ approaches zero and for $R(t)<0.05$ it is considered to be acceptable. In our experiments stress equilibrium was easy to obtain 

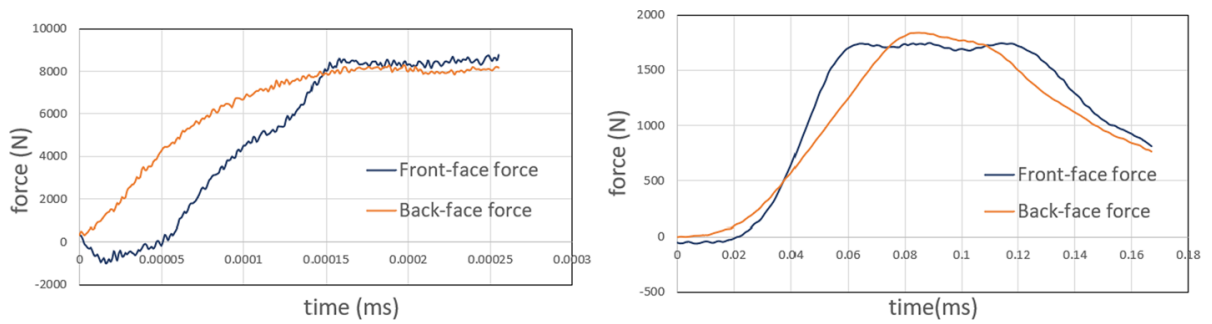

Fig. 3 Full history of front and back forces of a sample in aluminum bar (left) and PMMA bar (right), start of the stress equilibrium can be clearly observed in each setup

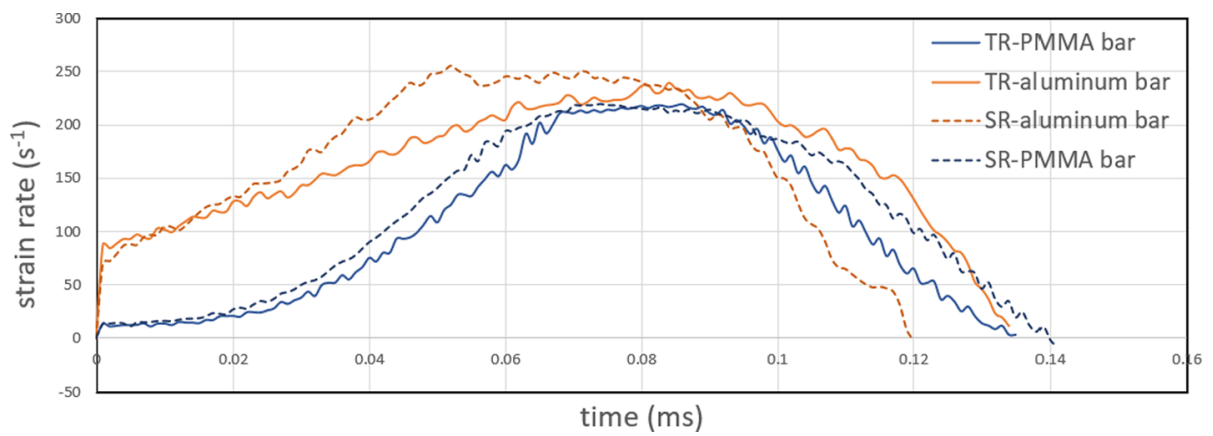

Fig. 4 Full strain rate history of SR and TR samples in aluminum and PMMA bars

for the PMMA bars but for aluminum bars it was more challenging. By applying a pulse shaper technique and also controlling the speed of the striker (Janiszewski et al. 2016), an acceptable stress equilibrium of $R(t)=0.04$ was achieved here. The full front and back force profiles of the specimens in aluminum and PMMA bars are illustrated in Fig. 3.

In addition to the two fundamental assumptions, friction and inertia effects are important issues during SHPB testing. In order to minimize friction for the specimens, the sample-bar contact surfaces were lubricated. Inertia has already been addressed by the design of the specimens, Sect. 2. The goal of SHPB testing is to determine the intrinsic material response and significant inertia effects can cause extra axial stresses, which blur the result (Chen and Song 2011).

\subsection{Pulse shaping}

For a well-defined strain rate in a SHPB test, the ideal incident pulse needs to rise to its maximum with a long rise time. To shape the wave pulse, thin cylindrically shaped lead plates with the thickness of $0.3-0.5 \mathrm{~mm}$ were fixed with grease at the impacted end of the aluminum incident bar. These pulse shapers can be plastically deformed, which enables a constant strain rate and also diminishes the oscillations of the wave (Weinberg and Khosravani 2018; Dai and Huang 2010). The full strain rate history of two SR and TR samples are shown in Fig. 4. The nearly constant strain rate for about $0.05 \mathrm{~ms}$ can be observed for these tested resins.

In the PMMA SHPB setup the incident strain waves are smoother. So it is not necessary to apply a pulse shaper to the incident bar surface. 
Fig. 5 Measured and corrected signal at the center of the two PMMA bars

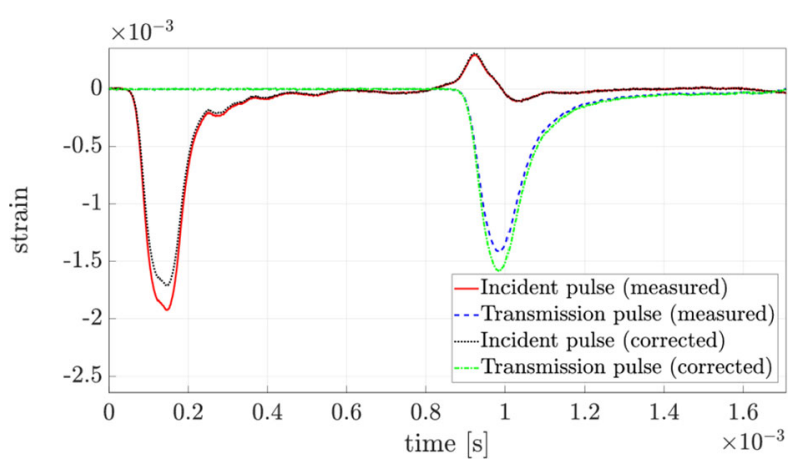

\subsection{Signal correction}

In PMMA bars wave scattering is reduced but the viscosity of the polymeric materials requires additional effort for data acquisition. The pulse measured at the strain gauge appliqued in the middle of the bar is not necessarily the same as the pulse entering the specimen and so an adaption of the signal is needed. Therefore, preparatory experiments analyzing the wave propagation in a single PMMA bar were conducted. After striker impact the back and forth traveling pulse is measured. Since the ends of the bar are free, the complete impulse is reflected but the amplitude decreases exponentially with time. Thus, an exponential function was fitted to the amplitude $A(x)$ of the measured signal and the free coefficients are adapted,

$$
A(x)=a e^{b x}+c e^{d x} .
$$

Here $x$ is the distance the wave moves through the material. The coefficients of Eq. (10) are $a=1.656 \cdot 10^{-3}, b=-0.2166 \cdot 10^{-3}, c=0.4267 \cdot 10^{-3}$ and $d=-0.04539 \cdot 10^{-3}$ for our SHPB setup and striker velocity. Other setups and specifications require a new calibration of these damping coefficients.

Based on this fit the signal at the bar-specimen interface can be estimated. For the measured incident pulse $\epsilon_{I}(t)$ the amplitude has to be corrected downwards, while for the measured reflected pulse $\epsilon_{T}(t)$ and transmitted pulse $\epsilon_{R}(t)$ the amplitude has to be corrected upwards. The corrected signal is then used to evaluate the SHPB equations (5)-(7).

The curve displayed in Fig. 5 shows the measured signal before and after correction. The primitive signal correction is working well for our study because the PMMA bars does not significantly change the shape or length of the wave. This approach is verified with numerical calculations in Sect. 4. A more elaborate method of signal correction is covered in another study by the authors Bieler and Weinberg (2020).

\section{Experimental results}

SHPB experiments need to be conducted multiple times in order to obtain reproducible results for wave shape, stress equilibrium and deduced values. Here, eight specimens of each resin material of Table 1 were evaluated in both experimental setups. In order to obtain the intrinsic behavior of the resin, each sample was tested only once. 


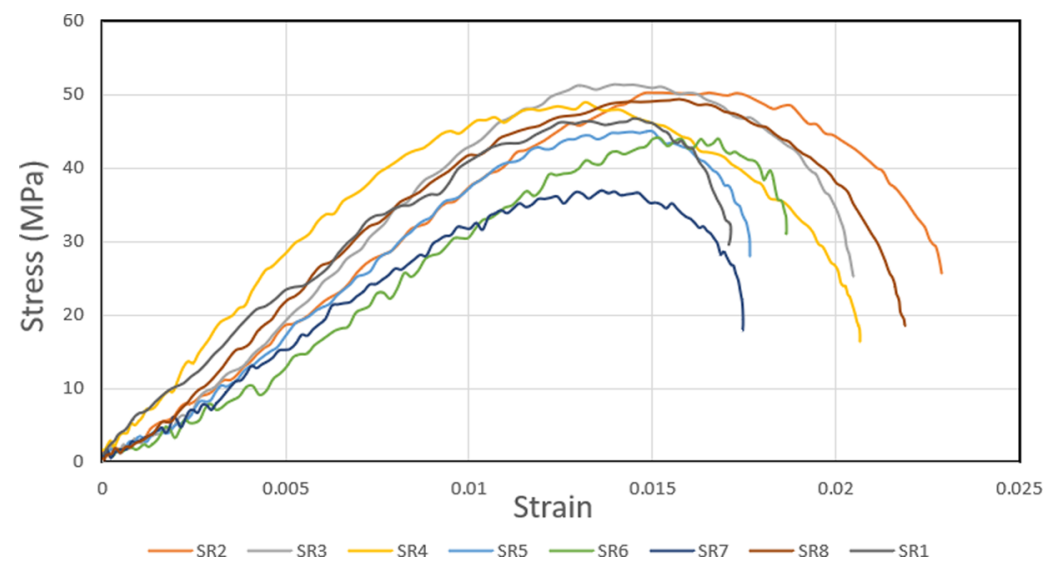

Fig. 6 Measured stress-strain curves of eight SR samples in the aluminum SHPB setup

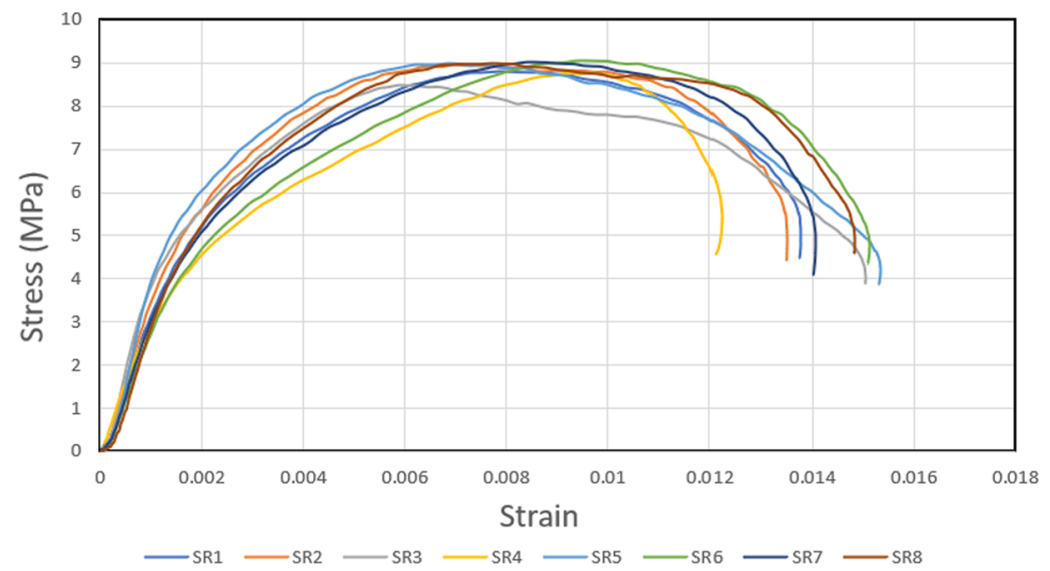

Fig. 7 Measured stress-strain curves of eight SR samples in the PMMA SHPB setup

\subsection{Aluminum bars}

In the aluminum SHPB setup the striker hits the incident bar with a velocity of about $10 \mathrm{~m} / \mathrm{s}$. This leads to a maximum stress of about $50 \mathrm{MPa}$ in both groups of specimens; see Fig. 6 and Fig. 8. Using Eq. (8) and a regression method for the precise slope calculation of the stress-strain curves, we deduced a mean dynamic elastic modulus of $3.7 \mathrm{GPa}$ for the SR and $3.4 \mathrm{GPa}$ for TR. It should be noted that for the regression only the linear part of the stress- strain curves (up to $0.5 \%$ strain) is considered. The strain rate is always in the range of $200-250 \mathrm{~s}^{-1}$. The values of the dynamic elastic modulus are significantly higher than the static modulus given by the manufacturer, which indicates a strain rate dependency in the polymeric resins.

\subsection{PMMA bars}

The signals measured with the PMMA bars are corrected as described above. Here the maximum stress in the specimen is approximately $9 \mathrm{MPa}$ and significantly lower than in the 

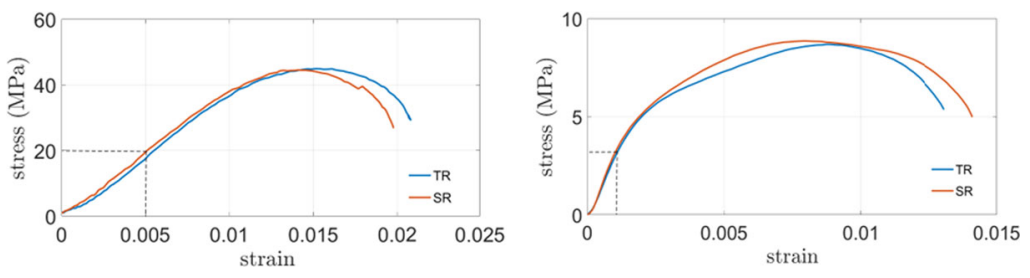

Fig. 8 Mean stress-strain curves for both groups of specimen tested in the aluminum bar setup (left) and in the PMMA bar setup (right); the box indicates the evaluated strain range

Table 3 Experimental results for standard resin (SR) and tough resin (TR)

\begin{tabular}{llllllll}
\hline Resin & \multicolumn{3}{l}{ Aluminum SHPB } & & \multicolumn{3}{l}{ PMMA SHPB } \\
\cline { 2 - 3 } & $\begin{array}{l}\text { striker vel. } \\
{\left[\frac{\mathrm{m}}{\mathrm{s}}\right]}\end{array}$ & $\begin{array}{l}\text { mean } \dot{\epsilon} \\
{\left[\mathrm{s}^{-1}\right]}\end{array}$ & $\begin{array}{l}E_{\mathrm{dyn}} \\
{[\mathrm{GPa}]}\end{array}$ & & $\begin{array}{l}\text { striker vel. } \\
{\left[\frac{\mathrm{m}}{\mathrm{s}}\right]}\end{array}$ & $\begin{array}{l}\text { mean } \\
{\left[\mathrm{s}^{-1}\right]}\end{array}$ & $\begin{array}{c}E_{\text {dyn }} \\
{[\mathrm{GPa}]}\end{array}$ \\
\hline SR & 10 & 236 & $3.7 \pm 0.9$ & 10 & 215 & $3.8 \pm 0.4$ \\
TR & 10 & 250 & $3.4 \pm 1.3$ & 10 & 207 & $3.4 \pm 0.9$ \\
\hline
\end{tabular}

previous setup; see Fig. 7. The reduced stress is related to the smaller elastic modulus of the PMMA but it does not play a role for deriving the dynamic elastic modulus of the resins, since only the stress-strain slope is evaluated.

The behavior of the resin specimens is almost the same. Again, regression is used and only the linear part of the stress-strain curves (up to $0.15 \%$ strain) is evaluated. For SR, a mean elastic modulus of $3.8 \mathrm{GPa}$ is obtained, whereas for TR the mean is $3.4 \mathrm{GPa}$. All obtained values and their standard deviations are summarized in Table 3.

\subsection{Numerical simulations of the SHPB setups}

In order to evaluate the effect of the two different SHPB setups we simulate the experiment by means of a finite element (FE) analysis with the commercial program Abaqus (Abaqus Standard user manual 2019). The main purpose of the simulation is to validate the strain wave propagation assumptions and to evaluate the corresponding wave signals.

The FE model was built for the full SHPB arrangement. All components are isotropic linear-elastic and meshed with three-dimensional 8 node linear brick elements, Fig. 9. The boundary conditions are chosen so that only longitudinal movement is allowed and at the interfaces surface to surface contact was defined. We use an explicit time integration (Abaqus explicit).

Parametric studies have been performed with strikers of $100 \mathrm{~mm}$ length and a predefined velocity of $10 \mathrm{~m} / \mathrm{s}$. The damping of the PMMA bars is modeled with a Rayleigh damping. For the aluminum bar a pulse shaper was added by a contact to contact surface. However, because the plastic properties are not well known, we had to reduce the impact speed to recover the incident wave. Figure 10 shows exemplarily the incident pulse propagation in aluminum. Although a slight discrepancy between simulation and experiment still remains, the pulse can be recovered quite accurately.

Figure 11 shows the incident wave pulse in a PMMA bar. For the experiment, the mean of the strain waves from the eight tested samples of each group is plotted. Here also exists good agreement between the experiment and simulation waves. 


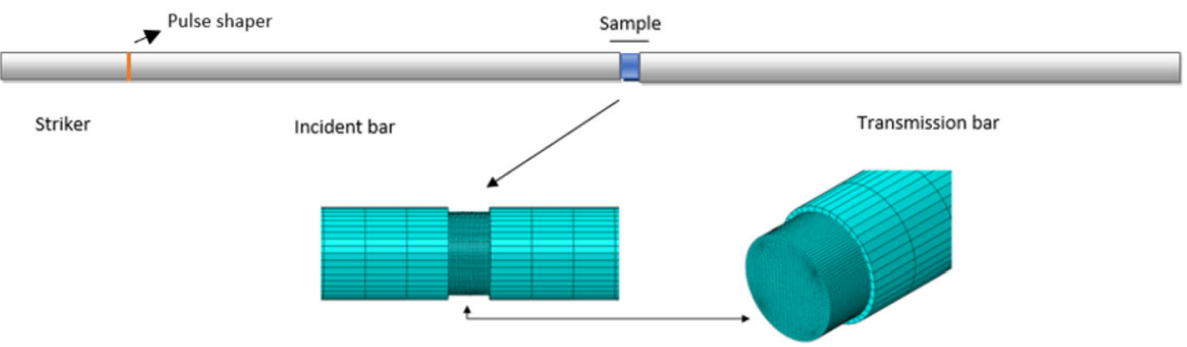

Fig. 9 FE-Mesh configuration of the SHPB setup

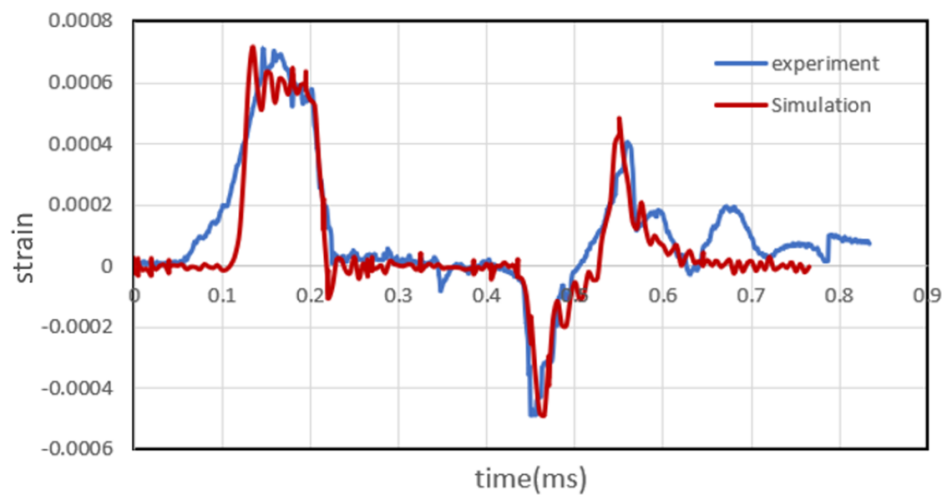

Fig. 10 Incident pulse propagation history in the aluminum SHPB setup

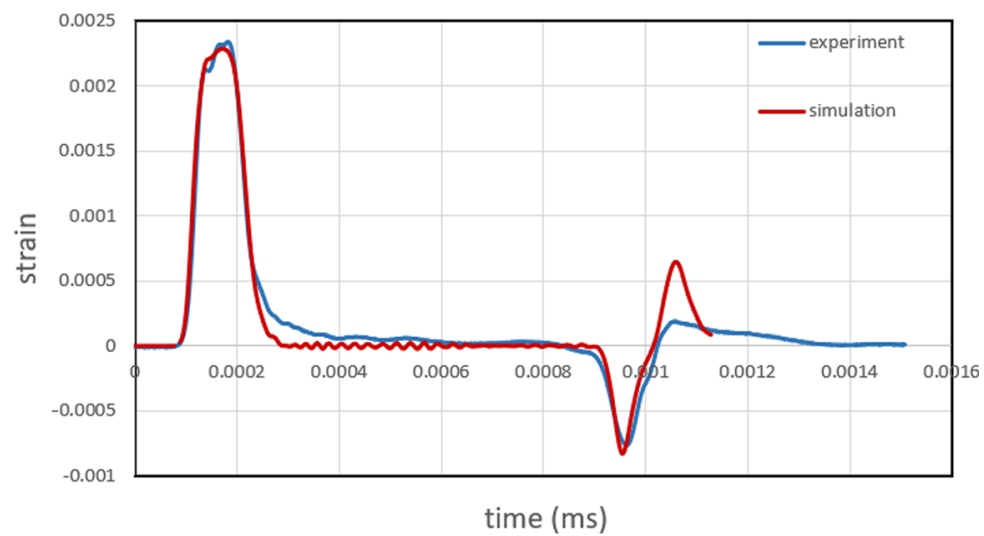

Fig. 11 Incident pulse propagation history in the PMMA SHPB setup

In Figs. 12 and 13 the transmitted pulse is shown for both setups. Again we observe a good agreement between the strain waves, although there are slight differences in some parts, which can be caused by several reasons, mainly related to the experimental condition. Moreover, mesh convergence studies were performed by implementing two different mesh densities (total number of elements 80976 [used], and 289576), and the represented 


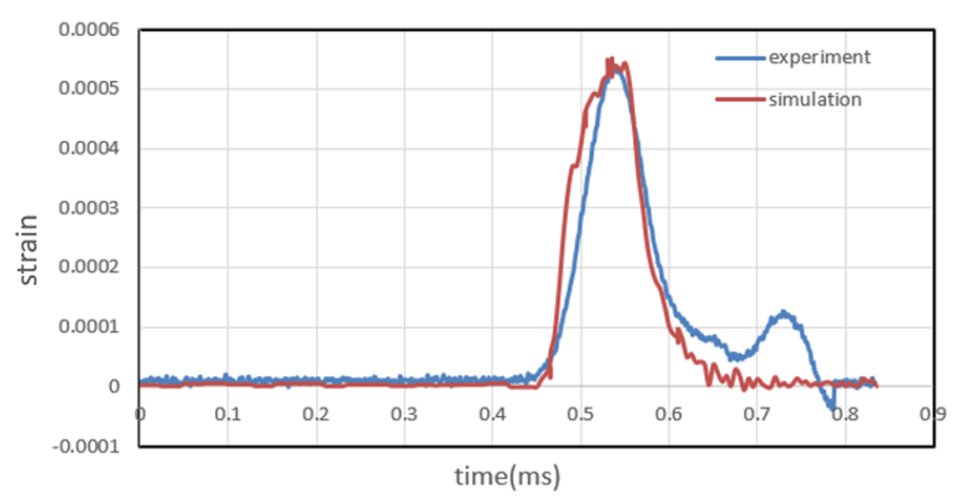

Fig. 12 Transmitted pulse propagation history in the aluminum SHPB setup with a TR sample

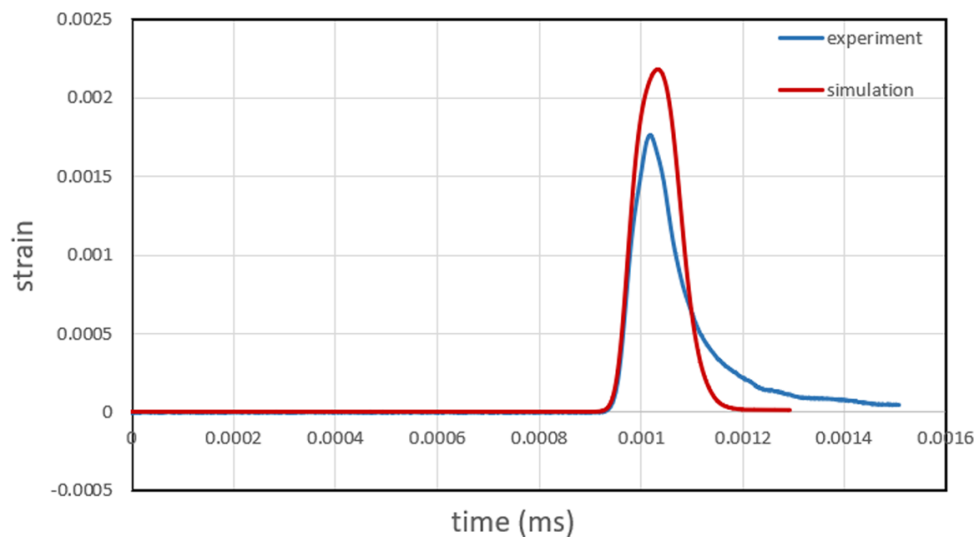

Fig. 13 Transmitted pulse propagation history in the PMMA SHPB setup with a TR sample

numerical simulation results were compared and prove the convergence. In general, it is recommended to consider the fact that the numerical results are much more reproducible than the experiments, as the conditions of the shots vary during the tests. Hence, during this study, we could validate the assumption of linear-elastic specimens, and the general improvement obtained by fit (10).

\subsection{Discussion}

SHPB experiments take a lot of attempts to obtain the best possible waves and the final outcome. Certainly, utilizing different bar materials changes the test conditions and the experimental details such as pulse shaping and data acquisition need to be adapted.

The waves gained from aluminum bars required pulse shaping in order to obtain a constant strain rate and to reduce dispersion, while there is little need for pulse shaping in the PMMA bar setup. Due to the inherent damping of PMMA, the strain wave shows almost no scattering but needs an amplitude signal correction. Further, the stress equilibrium is achieved more conveniently by using PMMA bars.

The FE analysis confirms the assumptions of the experiment and reproduces the waves nicely. Also the wave speed in the bars corresponds in the experiment and in the simulation. 

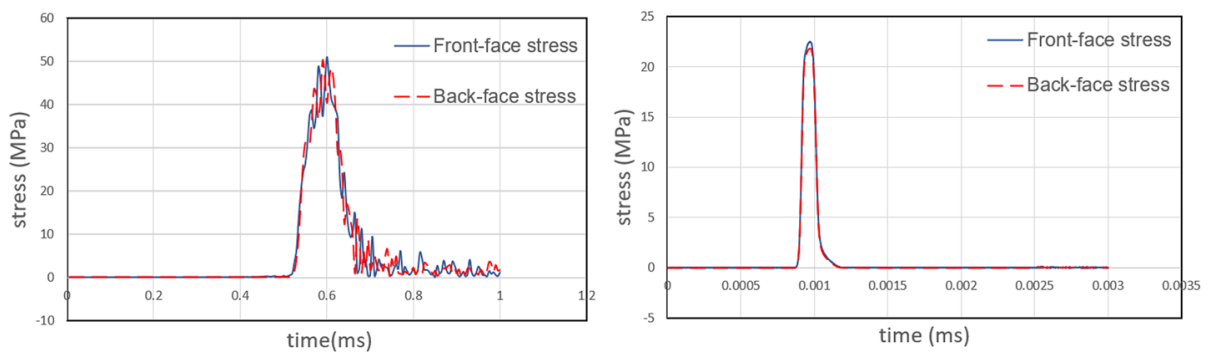

Fig. 14 Stress on both sides of the specimen in aluminum bar (left) and PMMA bar (right)

For instance, the experimental wave speed in the aluminum bar is $5015 \mathrm{~m} / \mathrm{s}$ and in the $\mathrm{FE}$ model it is $5000 \mathrm{~m} / \mathrm{s}$, which is another indication of the good agreement between the test and simulation. In addition, the stress on both sides of the specimen in simulation is shown in Fig. 14. Of course, some differences remain and are likely due to the condition of the test such as non-ideal contact. In total, utilizing the PMMA bars requires more efforts during and after the test, although less scattering in the prorogated waves and less varying results are obtained.

\section{Conclusion}

The vast applications of resins, specifically for 3D printing, has motivated us to investigate their dynamic behavior under high strain rates. Samples of standard and tough resin were tested in two different split Hopkinson pressure bar setups, one with aluminum and one with PMMA bars. In the PMMA bar setup the incident strain waves are smoother but have a lower amplitude than in the aluminum bars.

In our experiments we obtained mean dynamic elastic moduli of $3.7 \mathrm{GPa}$ for standard and 3.4 GPa for tough resin with aluminum bars. With PMMA bars, mean dynamic elastic moduli of $3.8 \mathrm{GPa}$ for standard and $3.4 \mathrm{GPa}$ for tough resin were obtained. The corresponding strain rates are $200-250 \mathrm{~s}^{-1}$. The dynamic values are about one-third higher than the corresponding static moduli of 2.8 and $2.7 \mathrm{GPa}$ for SR and TR samples, respectively. Clearly, both setups give a very similar dynamic response of the specimen.

In parallel to the experiment, finite element simulations were conducted to verify the tests. The computed waves are in good agreement with the experimental results. This allows us to conclude that PMMA incident and transmission bars are recommended for the dynamic testing of resin specimens.

Funding Note Open Access funding enabled and organized by Projekt DEAL.

\section{Declarations}

Conflict of interest On behalf of all authors, the corresponding author states that there is no conflict of interest.

Open Access This article is licensed under a Creative Commons Attribution 4.0 International License, which permits use, sharing, adaptation, distribution and reproduction in any medium or format, as long as you give appropriate credit to the original author(s) and the source, provide a link to the Creative Commons licence, and indicate if changes were made. The images or other third party material in this article are included in the article's Creative Commons licence, unless indicated otherwise in a credit line to the material. If material is not included in the article's Creative Commons licence and your intended use is not permitted by statutory regulation or exceeds the permitted use, you will need to obtain permission directly from the copyright holder. To view a copy of this licence, visit http://creativecommons.org/licenses/by/4.0/. 


\section{References}

Abaqus Standard user manual, Dessault Systems (2019)

Bieler, S., Weinberg, K.: Correction of Wave Signals for PMMA Split Hopkinson Pressure Bar Setups. PAMM 20(1), 202000143 (2020)

Bonyar, A., Santha, H.: 3D Rapid Prototyping Technology (RPT) as a powerful tool in microluidic development. Proc. Eng. 5, 291-294 (2010)

Chen, W., Song, B.: Split Hopkinson Bar, Design, Testing and Application. Springer, Berlin (2011)

Chen, W., Zhou, B.: Constitutive behavior of epon 828/T-403 at various strain rates. Mech. Time-Depend. Mater. 2, 103-111 (1998)

Dai, F., Huang, S.: Some fundamental issues in dynamic compression and tension tests of rock using split Hopkinson pressure bar. Rock Mech. Rock Eng. 43, 657-666 (2010)

Ferracane, L., Pfeifer, S.: Microstructural features of current resin composite materials. Curr. Oral Health Rep. 1, 205 (2014)

Formlabs. https://formlabs.com/de. Accessed 22.02.2020

Gorham, D.A.: Specimen inertia in high strain-rate compression. J. Phys. D, Appl. Phys. 22(12), 1888 (1989)

Hong, $\mathrm{H}$., Hu, M.: Dynamic mechanical behavior of hierarchical resin honeycomb by 3D printing. Polymers 13(1), 19 (2021)

Hopkinson, B.: A method of measuring the pressure produced in the detonation of high explosives or by the impact of bullets. In: Proceedings of the Royal Society of London, pp. 437-456 (1914)

Jager, S., Balthazard, R.: Dynamic thermo-mechanical properties of various flowable resin composites. J. Clin. Exp. Dent. 8(5), 534-539 (2016)

Jandt, D., Sigusch, W.: Future perspectives of resin-based dental materials. Dent. Mater. 25(8), 1001-1006 (2009)

Janiszewski, J., Buzantowicz, W., Baranowski, P.: Correction procedure of wave signals for a viscoelastic split Hopkinson pressure bar. Probl. Mechatron. Armament Aviat. Saf. Eng. 7(1), 17-30 (2016)

Kolsky, H.: Stress Waves in Solids. Dover Books on Physics, New York (1963)

Marghalani, Y.: Resin-Based Dental Composite Materials. Springer, Cham (2016)

Owens, A., Tippu, H.: A tensile split Hopkinson bar for testing particulate polymer composite under elevated rates of loading. Exp. Mech. 49, 799-811 (2009)

Ravichandran, G., Subhash, G.: Critical appraisal of limiting strain rates for compression testing of ceramics in a split Hopkinson pressure bar. J. Am. Ceram. Soc. 77, 263-267 (1994)

Roberson, D., Perez, A.: Comparison of stress concentrator fabrication for 3D printed polymeric izod impact test specimens. Addit. Manuf. 7, 1-11 (2015)

Rua, J., Buchely, M.: Structure-Property Relation of Epoxy Resin with Fique Fibers: Dynamic Behavior Using Split-Hopkinson Pressure Bar and Charpy Tests, Green Materials Engineering. The Minerals. Metals \& Materials Series. Springer, Cham (2019)

Ruamario, J., Sergio, F.: Structure-Property Relation of Epoxy Resin with Fique Fibers: Dynamic Behavior Using Split-Hopkinson Pressure Bar and Charpy Tests: Minerals. Metals and Materials Series. Springer, Cham (2019)

Sabbagh, J., Leloup, G.: Dynamic and static moduli of elasticity of resin based materials. Dent. Mater. 18, 64-71 (2002)

Weinberg, K., Khosravani, M.R.: On the tensile resistance of UHPC at impact. Eur. Phys. J. Spec. Top. 227, $167-177$ (2018)

Weinberg, K., Reza Khosravani, M., Thimm, B., Reppel, T., Bogunia, L., Aghayan, S., Nötzel, R.: Hopkinsonbar experiments as a method to determine impact properties of brittle and ductile materials. GAMMMitt. 41(2), 1-15 (2018)

Whiting, R., Jacobsen, P.H.: Dynamic mechanical properties of resin-based filling materials. J. Dent. Res. 59(1), 55-60 (1980)

Zhao, J., Knauss, W.: Applicability of the time-temperature superposition principle in modeling dynamic response of a polyurea. Mech. Time-Depend. Mater. 11, 289-308 (2007)

Zhao, X., Wang, S.: Thermal, Mechanical Properties and Morphology of Epoxy Resins Modified with Light Pyrolysis Natural Rubber. Wiley, New York (2017)

Publisher's Note Springer Nature remains neutral with regard to jurisdictional claims in published maps and institutional affiliations. 